\title{
Scheduling of Multicast Traffic in Tunable-Receiver WDM Networks with Non-Negligible Tuning Latencies
}

\author{
Zeydy Ortiz George N. Rouskas Harry G. Perros
}

TR-97-01

January 31, 1997

\begin{abstract}
We consider the problem of supporting multipoint communication at the media access control (MAC) layer of broadcast-and-select WDM networks. We first show that bandwidth consumption and channel utilization arise as two conflicting objectives in the design of scheduling algorithms for multicast traffic in this environment. We then present a new technique for the transmission of multicast packets, based on the concept of a virtual receiver, a set of physical receivers which behave identically in terms of tuning. We also show that the number $k$ of virtual receivers naturally captures the tradeoff between channel utilization and bandwidth consumption. Our approach decouples the problem of determining the virtual receivers from the problem of scheduling packet transmissions, making it possible to employ existing scheduling algorithms that have been shown to successfully hide the effects of tuning latency. Consequently, we focus on the problem of optimally selecting the virtual receivers, and we prove that it is $\mathcal{N} \mathcal{P}$-complete. Finally, we present four heuristics of varying degrees of complexity for obtaining virtual receivers that provide a good balance between the two conflicting objectives.
\end{abstract}

Keywords: Optical WDM Networks, Multipoint Communication, Multicast Traffic Scheduling

Department of Computer Science

North Carolina State University

Raleigh, NC 27695-8206 